\title{
Checklist helps reduce surgical complications, deaths*
}

\author{
News Release
}

\section{Surgical adverse events reduced by one third in trials in eight countries}

14 January 2009 - Hospitals in eight cities around the globe have successfully demonstrated that the use of a simple surgical checklist, developed by WHO, during major operations can lower the incidence of surgery-related deaths and complications by one third.

The studies were undertaken in hospitals in each of the six WHO regions. Analysis shows that the rate of major complications following surgery fell from $11 \%$ in the baseline period to $7 \%$ after introduction of the checklist, a reduction of one third. Inpatient deaths following major operations fell by more than $40 \%$ (from $1.5 \%$ to $0.8 \%$ ).

"The concept of using a brief but comprehensive checklist is surprisingly new to us in surgery. Not everyone on the operating teams were happy to try it. But the results were unprecedented. And the teams became strong supporters", said Dr Atul Gawande, main author of the study and team leader for the development of the WHO surgical safety checklist.

Data was collected from 7688 patients - 3733 before and 3955 after the checklist was introduced.

The study was carried out in hospitals in both high- and lower-income settings - in Ifakara (Tanzania), Manila (Philippines), New Delhi (India), Amman (Jordan), Seattle (United States of America), Toronto (Canada), London (United Kingdom) and Auckland (New Zealand). The reductions in complications proved to be of equal magnitude in high- and lower-income sites in the study.

\section{Implications for other medical fields}

"These findings have implications beyond surgery, suggesting that checklists could increase the safety and reliability of care in numerous medical fields", Dr Gawande said. "The checklists must be short, extremely simple, and carefully tested in the real world. But in specialties ranging from cardiac care to paediatric care, they could become as essential in daily medicine as the stethoscope".

The safe surgery checklist, which was launched by WHO as a recommended guideline for safe practice last year, has since gained global recognition by operating theatre staff, including surgeons and anaesthetists.

\footnotetext{
*Available from URL: http://www.who.int/mediacentre/news/releases/2009/safe_surgery_20090114/en/index.html (URL last accessed on August 11, 2009).
} 
It requires only a few minutes to complete at three critical points during operative care - before anaesthesia is administered, before skin incision and before the patient leaves the operating room. It is intended to ensure the safe delivery of anaesthesia, appropriate prophylaxis against infection, effective teamwork by the operating room staff and other essential practices in perioperative care.

"The immediate response to the checklist has been remarkable, and the studies undertaken in the pilot hospitals are significant. They will make a major contribution towards our goal of having 2500 hospitals around the world using the safe surgery checklist by the end of this year", said Sir Liam Donaldson, Chair of the WHO World Alliance for Patient Safety and Chief Medical Officer for England.

The results of the study are published on the web site of the New England Journal of Medicine. The material has appeared in the journal's printed issue on 29 January 2009. 